\title{
Comparison between high precision precession models for the ecliptic and the equator
}

\author{
N. Capitaine ${ }^{1}$, P. T. Wallace ${ }^{2}$, and J. Chapront ${ }^{1}$ \\ 1 Observatoire de Paris, SYRTE/UMR8630-CNRS, 61 avenue de l'Observatoire, 75014 Paris, France \\ ${ }^{2}$ H.M. Nautical Almanac Office, Space Science and Technology Department, CLRC / Rutherford Appleton Laboratory, UK \\ e-mail: ptw@star.rl.ac.uk
}

Received 23 December 2003 / Accepted 2 March 2004

\begin{abstract}
Three independent high precision solutions for precession were published in 2003 that provide expressions consistent with the IAU 2000A precession-nutation model (Mathews et al. 2002) and offer a possible replacement for the precession component of IAU 2000A, with improved dynamical consistency and a better basis for future improvement. Each is based upon an improved model for the precession of the ecliptic and, with respect to the IAU 1976 precession, they all provide higherdegree terms in the polynomials for the precession angles of the equator. This paper compares the expressions for the basic parameters of the above solutions for precession both of the ecliptic and the equator and investigates the possible physical and computational reasons for their differences. This leads to a realistic evaluation of the accuracy of the solutions and provides estimated deficiencies in them. These studies have identified expressions for the ecliptic precession quantities that are accurate to about $0.05 \mathrm{mas} / \mathrm{cy}$ over a two-millennium interval centered on J2000 instead of the few mas/cy accuracy of the current IAU model. They have also provided the theoretical and experimental basis for future improvements in the precession of the equator.
\end{abstract}

Key words. astrometry - reference systems - ephemerides - celestial mechanics - standards

\section{Introduction}

The IAU 2000A precession-nutation model provided by Mathews et al. (2002) (denoted MHB in the following) was adopted in IAU 2000 Resolution B1.6 and implemented in the IERS Conventions 2003. The precession component of the IAU 2000 model consists only of corrections to the precession rates in longitude and obliquity of the IAU 1976 precession. Therefore, at the same time, this Resolution recommended the development of new expressions for precession consistent with IAU 2000A.

Recent papers by Bretagnon et al. (2003), Fukushima (2003), Capitaine et al. (2003) and Harada \& Fukushima (2004) have provided new expressions for precession consistent with the IAU 2000A precession-nutation model. Each includes an improved model for the precession of the ecliptic, benefiting from the most accurate JPL ephemerides (Standish 1998), and each offers improved dynamical consistency for the precession of the equator.

All of these solutions have benefited from a previous precession solution by Williams (1994), denoted W94 in the following, which provided improved expressions with respect to those of Lieske et al. (1977), denoted L77 in the following

Send offprint requests to: N. Capitaine, e-mail: capitaine@syrte.obspm.fr and corresponding to the IAU 1976 precession. The W94 solution for the precession of the ecliptic was that of Simon et al. (1994), the source of which is the VSOP87 analytical solution (Bretagnon \& Francou 1988), improved by the introduction of the IERS Standards 1992 planetary masses (McCarthy 1992). The W94 solution for the precession of the equator was derived by solving the differential precession equations based on theoretical evaluation of all the contributions to the precession rates and on a preliminary VLBI estimation of the precession rates.

Bretagnon et al. (2003) provided precession expressions (denoted B03 in the following) derived from the SMART97 theory of the rotation of a rigid Earth (Bretagnon et al. 1997) using the MHB observed precession rate in ecliptic longitude. The precession of the ecliptic is that of Simon et al. (1994) improved by the introduction of the IERS 1992 planetary masses. The equinox offset and obliquity of the ecliptic at J2000 was derived by estimating the constant rotations between the JPL numerical ephemerides DE405 (constructed in the ICRS) and the VSOP analytical theory.

The solution by Fukushima (2003) is based on a new precession formula for the ecliptic, expressed as quadratic polynomials for two angles which specify the direction of the orbital angular momentum of the Earth-Moon barycenter (EMB) in heliocentric coordinates. The coefficients of these polynomials were determined by a fit to the numerical ephemerides DE405 
for the period 1600-2200, after having subtracted 86 Fourier and 4 mixed secular terms by using a non-linear method for harmonic analysis; the fit was corrected a posteriori for a quadratic polynomial that approximates the "great inequality" term (see Sect. 3.3). This fit also provided the equinox offset and the mean obliquity of the ecliptic at J2000. Adopting these expressions for the planetary precession, the solution for the precession of the equator was obtained by means of a fit to VLBI data of polynomials for the precession angles of the equator together with the recent theory of nutation by Shirai \& Fukushima (2001) for a non-rigid Earth. The solution is denoted F03. The latest solution for planetary precession, provided by Harada \& Fukushima (2004), denoted here HF04, is similar to the F03 solution, except that the a posteriori correction for the long-period terms was not applied. This solution is provided only in the form of the quadratic polynomials for the two angles (obliquity and node) specifying the instantaneous orbital plane of the EMB around the Sun for the period from 1629 to 2169.

Capitaine et al. (2003) obtained their solution for the ecliptic by using the JPL ephemerides DE406 to improve the analytical theory VSOP87. Fitting over a 2000-year interval was used to improve the polynomial terms in the expression for the component of the EMB orbital angular momentum with respect to a fixed ecliptic. The solution uses the value for the mean obliquity of the ecliptic at J2000 as derived from a fit of the dynamical theory for the Moon to LLR observations (Chapront et al. 2002). The equinox offset in the GCRS was derived from this fit using VLBI Earth Orientation Parameters. The model for the precession of the equator was obtained by solving the dynamical precession equations based both on the most recent expressions for the theoretical contributions to precession (in W94) and on the MHB estimates of the precession rates. Proper account was taken of all the perturbing effects on the observed quantities for the final solution, denoted P03. In addition to this final P03 solution, a preliminary solution, denoted $\mathrm{P}_{3}$ prel, was provided for the equator that was derived by exactly the same procedure, but was based directly on the unmodified MHB estimates for the precession rates (see Sect. 4.3 for more detail). This $\mathrm{P} 03_{\text {prel }}$ solution makes it easier to make direct comparisons with respect to other solutions based on the MHB precession rates and will be used for a number of comparisons with the B03 and F03 solutions in what follows.

The purpose of this paper is to evaluate the differences in the above independent solutions for the precession of the ecliptic and the equator, in order to clarify the properties of the various solutions, their dynamical consistency and the various physical causes for their differences. This will allow us to provide a realistic evaluation of the accuracy of the solutions.

Table A.1 sets out the notations used in this paper for designating specific precession models with their corresponding references.

Note that the coefficients of the recent solutions are quoted (cf. Tables 1 and 6) to a number of digits corresponding to a numerical convention for the purpose of internal computations that does not necessarily imply that any given coefficient is known to the quoted accuracy. This convention will not be used in the comparison tests, the purpose of which is simply to

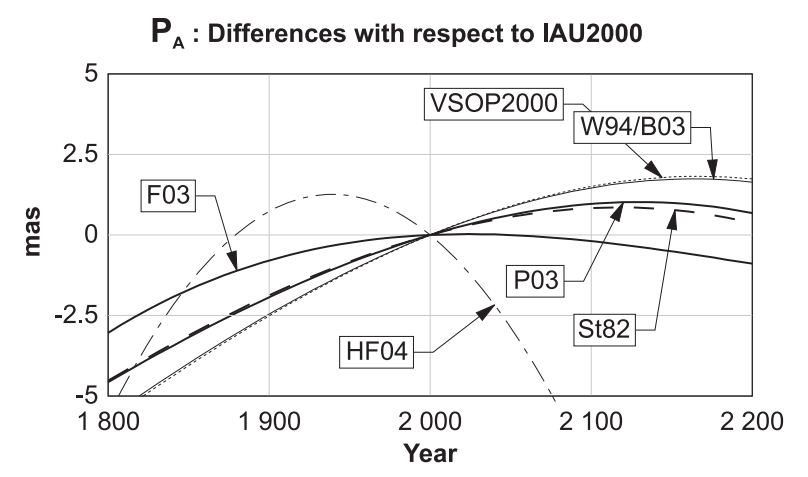

Fig. 1. Differences between various solutions for the precession of the ecliptic (B03: Bretagnon et al. 2003; F03: Fukushima 2003; HF04: Harada \& Fukushima 2004; P03: Capitaine et al. 2003; St82: Standish 1982; W94: Williams 1994; VSOP2000: Moisson \& Bretagnon 2001) with respect to the IAU 2000 solution: quantity $P_{\mathrm{A}}$.

\section{$Q_{A}$ : Differences with respect to IAU2000}

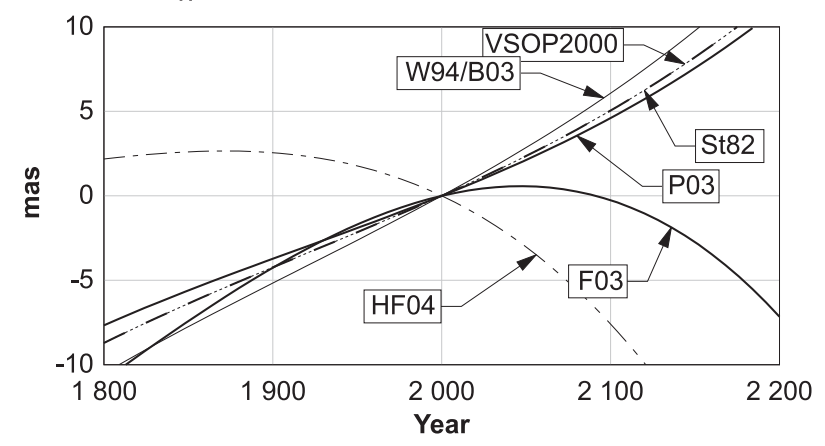

Fig. 2. Differences between various solutions for the precession of the ecliptic (see legend of Fig. 1) with respect to the IAU 2000 solution: quantity $Q_{\mathrm{A}}$.

evaluate the effects of different parameters on the solutions to a realistic precision.

\section{Comparison of the high precision precession solutions with IAU 2000}

Figures 1 to 4 show the differences of the various high precision solutions with respect to IAU 2000. Figures 1 and 2 are for the basic parameters $P_{\mathrm{A}}=\sin \pi_{\mathrm{A}} \sin \Pi_{\mathrm{A}}$ and $Q_{\mathrm{A}}=\sin \pi_{\mathrm{A}} \cos \Pi_{\mathrm{A}}$ that can be regarded as, respectively, the $x$ and $-y$ components of the secularly-moving ecliptic pole vector in the mean ecliptic frame at $\mathbf{J} 2000$. Note that the planetary theory VSOP87 introduces slightly different quantities, $p=(\sin \pi / 2) \sin \Pi$ and $q=(\sin \pi / 2) \cos \Pi$ (where $\pi$ is the inclination on the ecliptic and $\Pi$ the longitude of the ascending node); time polynomials for $P_{\mathrm{A}}$ and $Q_{\mathrm{A}}$ can easily be derived from the secular developments of $p$ and $q$.

Figures 3 and 4 are for the basic parameters, $\psi_{\mathrm{A}}$ and $\omega_{\mathrm{A}}$, that are, respectively, the precession in longitude and obliquity referred to the mean ecliptic at J2000 and therefore provide the 
$\psi_{\mathrm{A}}$ : Differences with respect to IAU2000

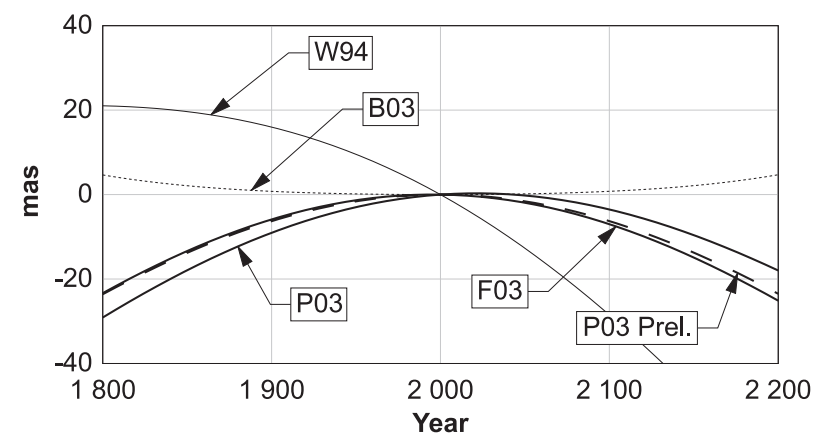

Fig. 3. Differences between various solutions for the precession of the equator (B03: Bretagnon et al. 2003; F03: Fukushima 2003; P03 and

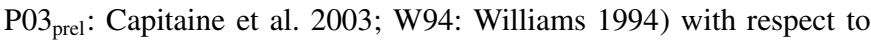
the IAU 2000 solution: quantity $\psi_{\mathrm{A}}$.

$\omega_{A}:$ Differences with respect to IAU2000

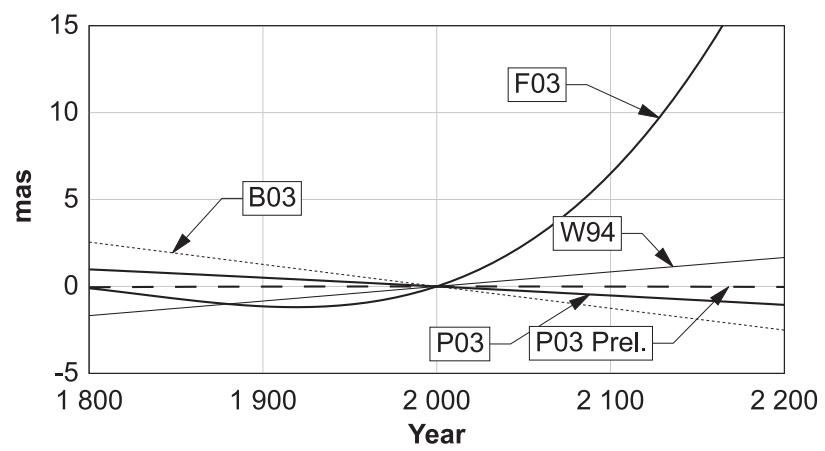

Fig. 4. Differences between various solutions for the precession of the equator (see legend of Fig. 3) with respect to the IAU 2000 solution: quantity $\omega_{\mathrm{A}}$.

orientation of the mean equator of the Celestial Intermediate Pole (CIP) ${ }^{1}$.

The plots for the ecliptic show, in Figs. 1 and 2, the W94, B03, F03 and P03 solutions. Three other solutions are plotted also:

- the preliminary analytical solution VSOP2000, described in Moisson \& Bretagnon (2001) but not yet published in a final form, reported in Bidart (2000) as $p$ and $q$ variables that we first converted into polynomial expressions for $P_{\mathrm{A}}$ and $Q_{\mathrm{A}}$;

- the new HF04 solution, originally provided as second degree polynomial fits to DE405 instantaneous node and obliquity that we first converted into polynomial expressions for $P_{\mathrm{A}}$ and $Q_{\mathrm{A}}$;

- a solution due to Standish (1982), denoted St82 in the following, that was originally provided as third degree polynomial fits to DE102 instantaneous node and obliquity, that we also converted into polynomial expressions for $P_{\mathrm{A}}$ and $Q_{\mathrm{A}}$. This solution was based on a method similar to

1 The CIP has been defined by IAU 2000 Resolution B1.7 as the intermediate pole separating nutation from polar motion explicitly at the 2-day period (i.e. nutations in space with periods less than 2 days being modeled by their equivalent polar motion with respect to the terrestrial frame). the one used subsequently in F03, but over a longer interval (1435 years) and with correction for the GI terms.

Note that the constant terms of the above $P_{\mathrm{A}}$ and $Q_{\mathrm{A}}$ polynomials provide the offsets to transform the solutions into the mean ecliptic frame.

The plots for the equator show, in Figs. 3 and 4, the W94, B03, F03, P03 and P03 prel solutions.

Regarding the precession of the ecliptic, we note the significant differences between all the recent solutions and the IAU 2000 solution, which is in fact the IAU 1976 solution, L77. We also note the good agreement between the W94, B03, VSOP2000, St82 and P03 solutions, differing only, at the level of precision provided by the figures, by a secular trend, whereas there are very large discrepancies with respect to the Fukushima 2003 solution (F03). We note that the discrepancies with respect to the Harada \& Fukushima 2004 solution (HF04) are even larger. Note that this is in agreement with the comparison provided in the HF04 paper which shows for this latest solution for the planetary precession differences of the order of 50 mas in the orientation angles with respect to the W94, B03 and St82 solutions over the 600-year interval of the HF04 fit.

Regarding the precession of the equator, we note the quadratic differences in $\psi_{\mathrm{A}}$ between the W94, P03, P03 $3_{\text {prel }}$ and F03 solutions and the IAU 2000 and the Bretagnon et al. (2003) solution (B03), these two last solutions being very similar at the level of precision provided by the Figures. We also note the very large discrepancy in $\omega_{\mathrm{A}}$ between the Fukushima (2003) solution (F03) and the others, which are all very close to the IAU 2000 solution. The use of the P03 $3_{\text {prel }}$ solution instead of P03 frees the comparisons from the secular difference coming from the different integration constants.

\section{Precession of the ecliptic}

\subsection{Comparison between the various solutions for the precession of the ecliptic}

The various expressions for the precession of the ecliptic, described in the previous section, are set out in Table 1. As previously mentioned, the IAU 2000 solution is unchanged with respect to IAU 1976, and we note that the recent solutions all provide higher degree developments and coefficients with a much higher resolution. We also note that the B03 solution is nearly the same as W94, but with an improved numerical precision. This is due to the fact that these two solutions are derived from a similar computation (as described in the introduction) whereas the B03 solution, being computed from the original expressions, achieves a better accuracy.

Comparison between the numerical values of the coefficients of the expressions for $P_{\mathrm{A}}$ and $Q_{\mathrm{A}}$ confirms (i) the significant difference between the Fukushima (2003) expressions (F03) and other expressions, reaching several mas/cy in the $t$ coefficient in $P_{\mathrm{A}}$ and resulting in very different values in the coefficients of degree higher than 2 for both quantities; (ii) the slight difference between the solutions P03 of Capitaine et al. (2003) and B03 of Bretagnon et al. (2003), of the order of 1 mas in the $t$ coefficient and much smaller (tens of $\mu$ as) in the coefficients of higher degree. 
Table 1. Comparisons between expressions for the ecliptic precession quantities (Sources: W94, Williams 1994; B03, Bretagnon et al. 2003; VSOP2000, Moisson \& Bretagnon 2001; F03, Fukushima 2003; P03, Capitaine et al. 2003; HF04, Harada \& Fukushima 2004; St82, Standish 1982); unit: milliarcsecond.

\begin{tabular}{|c|c|c|c|c|c|c|}
\hline Source & & $t$ & $t^{2}$ & $t^{3}$ & $t^{4}$ & $t^{5}$ \\
\hline IAU 1976 & & 4197.6 & 194.47 & -0.179 & & \\
\hline IAU 2000 & & 4197.6 & 194.47 & -0.179 & & \\
\hline W94 & & 4199.610 & 193.971 & -0.223 & -0.001 & \\
\hline B03 & $P_{\mathrm{A}}$ & 4199.604 & 193.9715 & -0.22350 & -0.001035 & +0.0000019 \\
\hline VSOP2000 & & 4199.653 & 193.9712 & -0.22366 & -0.001036 & +0.0000019 \\
\hline F03 & & 4197.822 & 193.9782 & -0.10053 & +0.000097 & -0.000003 \\
\hline $\mathrm{P} 03$ & & 4199.094 & 193.9873 & -0.22466 & -0.000912 & +0.0000120 \\
\hline HF04 & & 4193.477 & 191.1599 & -0.09908 & +0.000099 & \\
\hline St82 & & 4198.998 & 193.9518 & -0.22290 & +0.000196 & \\
\hline IAU 1976 & & -46815.0 & 50.59 & +0.344 & & \\
\hline IAU 2000 & & -46815.0 & 50.59 & +0.344 & & \\
\hline W94 & & -46809.560 & 51.043 & +0.522 & -0.001 & \\
\hline B03 & $Q_{\mathrm{A}}$ & -46809.550 & 51.0421 & +0.52228 & -0.000569 & -0.0000014 \\
\hline VSOP2000 & & -46809.979 & 51.0291 & +0.52229 & -0.000564 & -0.0000013 \\
\hline F03 & & -46812.649 & 48.3315 & -0.00879 & -0.000215 & +0.000039 \\
\hline $\mathrm{P} 03$ & & -46811.015 & 51.0283 & +0.52413 & -0.000646 & -0.0000172 \\
\hline HF04 & & -46819.720 & 48.0717 & -0.00861 & -0.000206 & \\
\hline St82 & & -46810.532 & 50.9973 & +0.51924 & -0.000206 & \\
\hline
\end{tabular}

In order to evaluate the accuracy of the solutions, we compared the various solutions with DE406 in Sect. 3.2 and investigated some possible causes for the differences. The effect of the long-period variations of the ecliptic in the determination of the polynomial part is considered in Sect. 3.3, the effect of the numerical ephemerides that were used for the fit of the polynomial in Sect. 3.4 and the inaccuracy in the secular term of the analytical ephemerides is evaluated in Sect. 3.5.

\subsection{Differences of models for the ecliptic with respect to the numerical ephemerides DE406}

The Earth-Moon barycenter orbital position and velocity as provided by the JPL DE406 numerical ephemerides can be used for computing a DE406 "ecliptic". The DE406 predictions can, as far as this exercise is concerned, be considered as a source of "observations". The accuracy of the models for the ecliptic can therefore be evaluated through their agreement with these observations.

For a better characterization of the solutions, complete analytical models for the ecliptic were considered in the comparisons with DE406, rather than relying on the non-secular terms "averaging out". The resulting models used the VSOP87 analytical solution for the non-polynomial part, which includes several hundred periodic terms with periods from a few days to 25770 years, but replaced the polynomial part of the VSOP87 analytical solution with a new "P03" polynomial. We have compared the differences between these complete models for the ecliptics and DE406 and those of the B03 and F03 solutions with respect to the $\mathrm{P} 03$ solution (i.e. DE406).

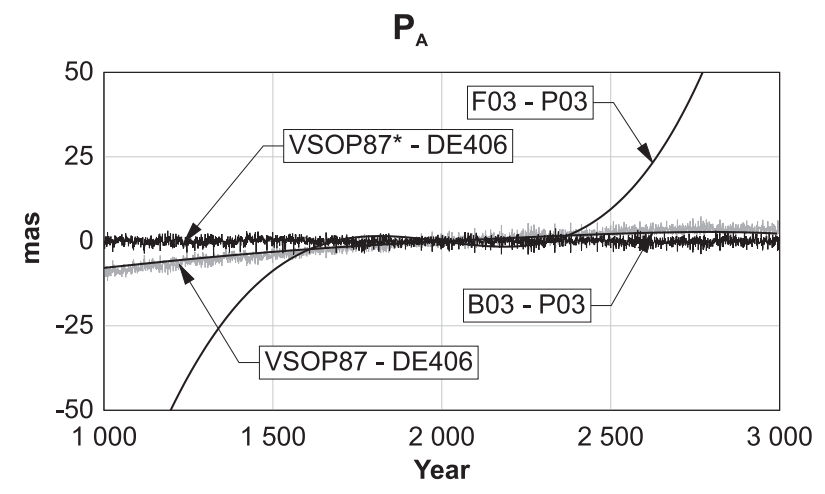

Fig. 5. Comparison of models for the ecliptic (quantity $P_{\mathrm{A}}$ ) with respect to DE406 over a 2000-year interval (F03: Fukushima 2003; B03: Bretagnon et al. 2003; P03: Capitaine et al. 2003; VSOP87: Bretagnon \& Francou 1988; VSOP87*: see (1)).

Figures 5 and 6 display the following differences over the years 1000-3000:

(1) VSOP87, with its original polynomials, minus DE406;

(2) VSOP87, with the adjusted polynomials (i.e. the P03 solution), denoted VSOP87* in the figures, minus DE406;

(3) B03-P03;

(4) F03-P03.

The above curves on the same diagram illustrate the following points:

(i) the curves (1) and (2) reveal the short-period "noise" produced by inaccuracy in the short-period terms of VSOP87; 


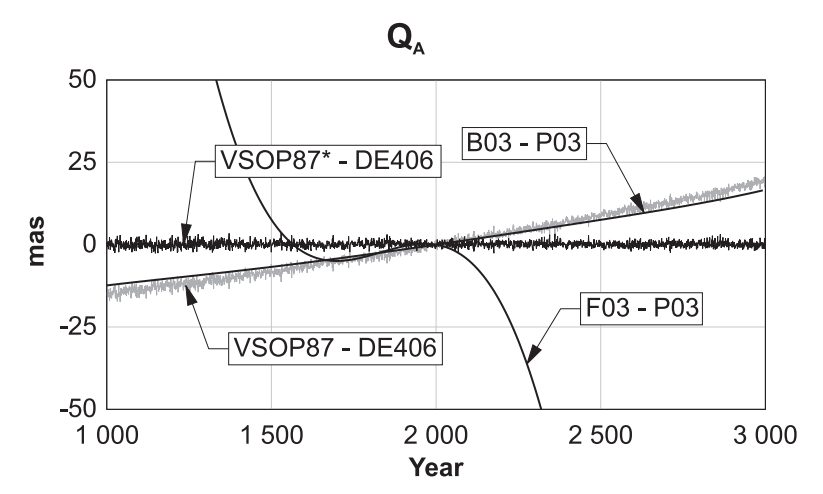

Fig. 6. Comparison of models for the ecliptic (quantity $Q_{\mathrm{A}}$ ) with respect to DE406 over a 2000-year interval (see legend of Fig. 5).

(ii) the curve (3) of the difference between the B03 solution of Bretagnon et al. 2003 and DE406 follows the curve of the difference between VSOP87 and DE406, with a slight change of slope which is due to the small change in the ecliptic model coming from the IERS 1992 improvement in the planetary masses;

(iii) the F03 solution of Fukushima (2003) diverges considerably from DE406 over a long time interval; this divergence is more spectacular than over a short time interval. It implies that the high-degree terms in the F03 secular developments fail seriously;

(iv) the P03 analytical approach of Capitaine et al. (2003) does not introduce any bias in the computation of $P_{\mathrm{A}}$ and $Q_{\mathrm{A}}$.

\subsection{Effect of long-period terms in the precession solution}

A possible explanation for the observed discrepancies in the F03 solutions of Fukushima (2003) is the method employed to discriminate between the secular motion of the ecliptic, represented by polynomial expressions, and periodic components, especially those of long period. This is even more critical in the case of the HF04 solution of Harada \& Fukushima (2004), computed without any correction for the long-period contributions. With this in mind, we have attempted to characterize the possible influence of these slow periodic variations on the fitting process. For this study, we retained the largest Fourier and Poisson terms in VSOP87 with periods greater than 500 years; their arguments are set out in Table 2.

Figures 7 and 8 show the contributions in $P_{\mathrm{A}}$ and $Q_{\mathrm{A}}$ (reaching \pm 20 mas) of the well-known $2 J-5 S$ "great inequality" (denoted GI) in the EMB motion, of 883-year period, and of very-long-period terms, over an interval covering 2000 years.

Concerning the very-long-period terms, it appears that the effects on the polynomial models are relatively small. Therefore, if we develop such terms with respect to time and introduce them in the secular polynomials for $P_{\mathrm{A}}$ and $Q_{\mathrm{A}}$, the results will be only slightly changed and the formulas for the precession of the ecliptic should not be seriously modified. It is only a question of the definition of "secular motion" related to the time interval, that has no significant influence on the model for the precession of the ecliptic but could result in
Table 2. The long-period terms in the VSOP87 model for $p, q$ : $A t^{\alpha} \cos (\omega t+\phi)$. The planetary and lunar arguments are $T=$ Earth, $M a=$ Mars, $J=$ Jupiter, $S=$ Saturn, $D=$ Delaunay's argument (the difference of the mean longitudes of Moon and Sun), $L_{\text {Moon }}=$ mean longitude of the Moon.

\begin{tabular}{lcccc}
\hline \hline Argument & $\alpha$ & $A(\mathrm{mas})$ & $\phi\left(^{\circ}\right)$ & $P=\frac{2 \pi}{\omega}(\mathrm{yr})$ \\
\hline Variable $: q$ & & & & \\
$2 J-5 S$ & 0 & 6.72 & 125.2 & 883 \\
$4 T-8 M a+3 J$ & 0 & 0.12 & 350.4 & 1783 \\
$2 J-5 S$ & 1 & 0.50 & 174.6 & 883 \\
$T+D-L_{\text {Moon }}$ & 1 & 0.17 & 180.0 & 25770 \\
\hline Variable $: p$ & & & & \\
$2 J-5 S$ & 0 & 7.57 & 30.4 & 883 \\
$2 J-5 S$ & 1 & 0.30 & 114.6 & 883 \\
$T+D-L_{\text {Moon }}$ & 1 & 0.17 & 270.0 & 25770 \\
\hline
\end{tabular}

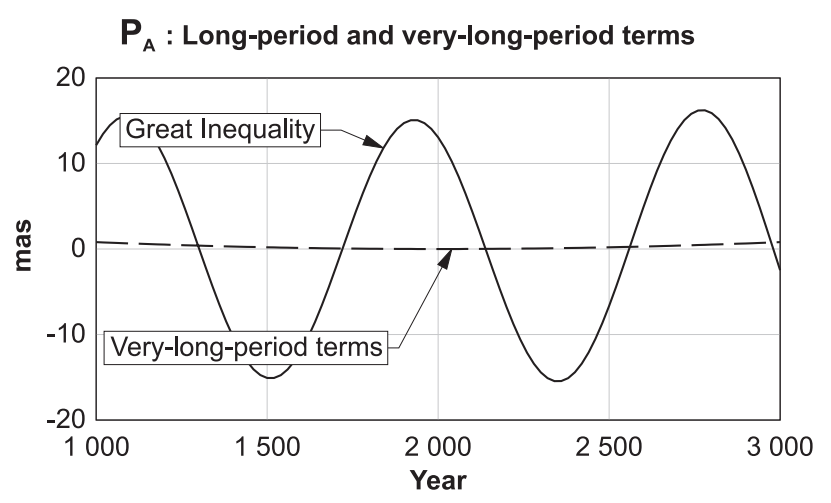

Fig. 7. Long-period variations in $P_{\mathrm{A}}$.

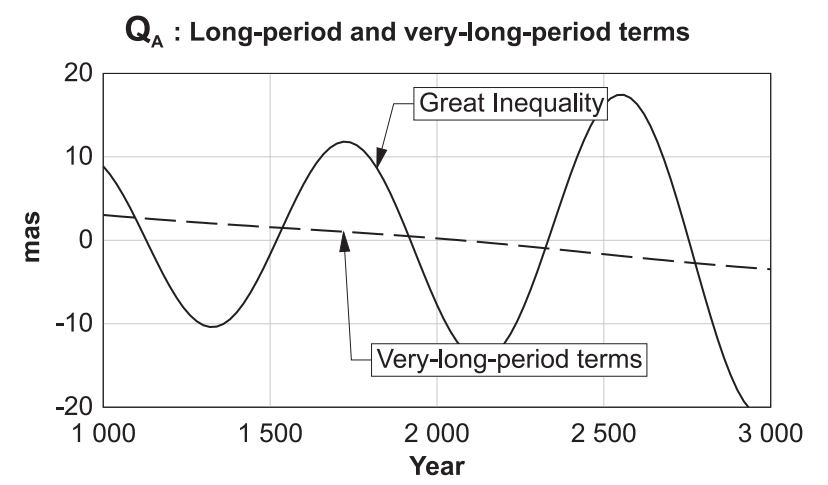

Fig. 8. Long-period variations in $Q_{\mathrm{A}}$.

discrepancies of the order of a few tenths of a milliarcsecond between solutions that do not use the same convention.

On the other hand, Figs. 7 and 8 show that polynomial fits over a few hundred years are an unsatisfactory way of allowing for the "great inequality", and this could explain the divergent behavior of the F03 solutions for $P_{\mathrm{A}}$ and $Q_{\mathrm{A}}$, as plotted in Figs. 5 and 6 (note that these cover the same time 2000-year interval as Figs. 7 and 8).

Table 3 provides comparisons between different ways of fitting a polynomial to the DE406 ecliptic, the last two lines of the Table being for the F03 and HF04 solutions. 
Table 3. Comparisons between expressions for the ecliptic precession quantities according to the way the determination has been made (Sources: P03, Capitaine et al. 2003; F03, Fukushima 2003; HF04, Harada \& Fukushima 2004); unit: milliarcsecond.

\begin{tabular}{|c|c|c|c|c|c|c|}
\hline Source & & $t$ & $\overline{t^{2}}$ & $\overline{t^{3}}$ & $\overline{t^{4}}$ & $\overline{t^{5}}$ \\
\hline (i.a) & & 4198.9 & 201.6 & -0.4 & -3.2 & -0.7 \\
\hline (i.b) & & 4197.2 & 193.7 & -0.1 & +0.0 & -0.0 \\
\hline (ii) & & 4204.3 & 205.0 & -0.8 & -3.4 & -0.7 \\
\hline (iii.a) & $P_{\mathrm{A}}$ & 4199.2 & 193.9 & -0.2 & +0.0 & +0.0 \\
\hline (iii.b) & & 4193.8 & 190.5 & +0.2 & +0.2 & +0.0 \\
\hline (iii.c) & & 4196.8 & 193.6 & -0.1 & +0.0 & -0.0 \\
\hline (iv) (P03) & & 4199.1 & 194.0 & -0.2 & -0.0 & +0.0 \\
\hline F03 & & 4197.8 & 194.0 & -0.1 & +0.0 & -0.0 \\
\hline HF04 & & 4193.5 & 191.2 & -0.1 & +0.0 & \\
\hline (i.a) & & -46820.8 & 52.3 & +2.3 & +0.5 & +0.0 \\
\hline (i.b) & & -46814.6 & 51.3 & +0.7 & -0.0 & -0.0 \\
\hline (ii) & & -46813.0 & 50.4 & +1.6 & +0.5 & +0.0 \\
\hline (iii.a) & $Q_{\mathrm{A}}$ & -46811.2 & 51.1 & +0.6 & -0.0 & -0.0 \\
\hline (iii.b) & & -46819.1 & 53.0 & +1.2 & -0.1 & -0.0 \\
\hline (iii.c) & & -46814.3 & 51.3 & +0.7 & -0.0 & -0.0 \\
\hline (iv) (P03) & & -46811.0 & 51.0 & +0.5 & -0.6 & -0.0 \\
\hline F03 & & -46812.6 & 48.3 & -0.0 & -0.0 & -0.0 \\
\hline HF04 & & -46819.7 & 48.1 & -0.0 & -0.0 & \\
\hline
\end{tabular}

The fits performed in the present work correspond to:

(i.a) a polynomial alone over a time interval of 600 years (1600-2200);

(i.b) a polynomial alone over a time interval of 2000 years (1000-3000);

(ii) a polynomial, plus the "great inequality" term, over a time interval of 600 years (1600-2200);

(iii.a) a polynomial representing the difference between the periodic component of the analytical solution VSOP87 and the DE406 ecliptic over a time interval of 600 years (1600-2200);

(iii.b) a polynomial representing the difference between the periodic component of the analytical solution VSOP87 and the DE406 ecliptic over a time interval of 600 years (1600-2200) with the Table 2 terms removed;

(iii.c) a polynomial representing the difference between the periodic component of the analytical solution VSOP87 and the DE406 ecliptic over a time interval of 2000 years (1000-3000) with the Table 2 terms removed;

(iv) a polynomial difference between the analytical solution VSOP87 and the DE406 ecliptic over a time interval of 2000 years (1000-3000; P03 solution).

The corresponding final DE405/406-to-ecliptic rotation angles are set out in Table 4.

Tables 3 and 4 show the non-negligible difference between the coefficients of the polynomial and the corresponding ecliptic rotation angles according to the way they are derived and the time interval for which the estimates are made, if an analytical solution including an accurate model of the long-period
Table 4. Rotation angles $\epsilon_{0}, \phi_{0}$ and $\psi_{0}$ in milliarcsecond (see description of the labels in the caption of Table 3 ).

\begin{tabular}{lccc}
\hline \hline Fit to DE406 & $\epsilon-23^{\circ} 26^{\prime} 21^{\prime \prime}$ & $\phi_{0}$ & $\psi_{0}$ \\
\hline (i.a) & 401.0 & 28.2 & 17.4 \\
(i.b) & 407.6 & 47.9 & 35.5 \\
(ii) & 408.8 & 61.1 & 47.6 \\
(iii.a) & 408.9 & 51.4 & 38.7 \\
(iii.b) & 401.4 & 18.5 & 08.5 \\
(iii.c) & 407.8 & 46.2 & 34.0 \\
(iv) (P03) & 408.9 & 51.3 & 38.6 \\
F03 & 411.3 & 52.4 & 45.2 \\
HF04 & 405.8 & 21.1 & \\
\hline
\end{tabular}

components is not used as a reference. This shows how a fit over a time interval of insufficient length can give significant inaccuracies in the polynomial determination.

We note in particular:

- the discrepancies in the higher-degree coefficients of solutions (i.a) and (ii) that result from fitting to DE406, over 600 years, either without correction for periodic terms (i.a), or for the GI term only (ii);

- significant changes in the solution according to the length of the interval (i.e. between (i.a) and (i.b) and (iii.b) and (iii.c)), the change being much more important in cases (i.a) and (i.b) where the solution is not corrected for periodic terms;

- significant changes in the solution for the same interval of fit when considering or not considering the very-long period terms (i.e. between (i) and (ii), or (iii.a) and (iii.b)); - the similarity between solutions (iii.b) and HF04.

Special tests have been introduced to characterize the error resulting from an approximation of the GI term by a quadratic polynomial, as used in F03. Figures 9 and 10 display together the differences F03-P03 and the residuals of the GI term with respect to its approximation with a polynomial of degree 2 over the interval of approximation (1600-2200) of the F03 solution for $P_{\mathrm{A}}$ and $Q_{\mathrm{A}}$, respectively. The notation "Delta GI" means the difference between the $2 J-5 S$ Fourier and Poisson terms as they are in the VSOP $P_{\mathrm{A}}$ and $Q_{\mathrm{A}}$ series, and the "parabolic fit" of these terms in the sense parabolic fit minus VSOP. Note that using all the terms of Table 2, instead of the GI term only, leads to very similar results. Such comparisons illustrate that discrepancy between the F03 and P03 solutions is in good agreement with the differences over 600 years between the GI and a parabolic fit. For $P_{\mathrm{A}}$ (Fig. 9), the F03-P03 curve roughly represents the mean (in the least squares approximation sense) of the two oscillations that are displayed by Delta GI in the 1600-2200 interval. In the case of $Q_{\mathrm{A}}$ (Fig. 10), the agreement is rather better. Figures 9 and 10 also show that the short-period terms (i.e. periodic terms with periods shorter than 500 years) were correctly removed in the F03 solution for $P_{\mathrm{A}}$ and $Q_{\mathrm{A}}$.

The tests described in this section provide a realistic estimation of the deficiencies in the F03 solution of Fukushima (2003) for the precession of the ecliptic in showing that, whereas 


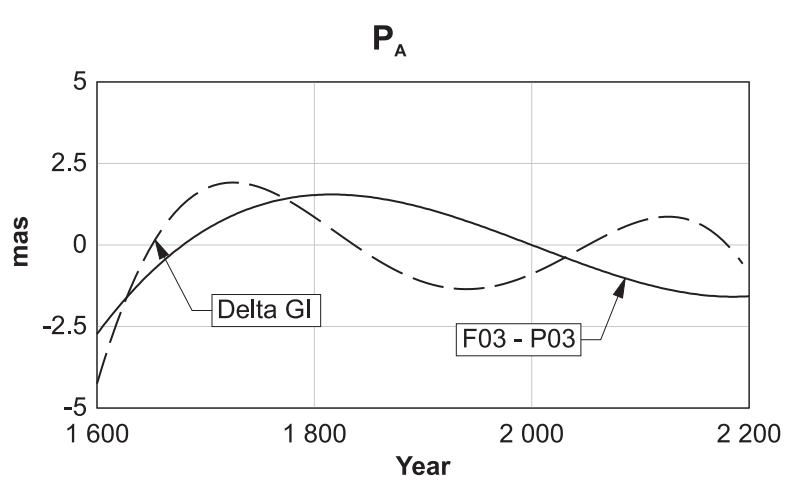

Fig. 9. Comparison between differences F03-P03 (F03: Fukushima 2003; P03: Capitaine et al. 2003) and the residuals of the "great inequality" term with respect to its approximation with a polynomial of degree 2 (in mas: $12.2-4.3 t-2.8 t^{2}$ ): quantity $P_{\mathrm{A}}$.

\section{$\mathbf{Q}_{\mathrm{A}}$}

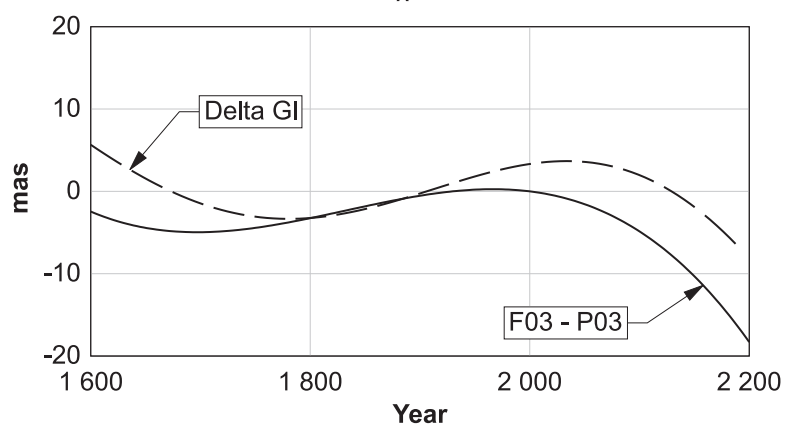

Fig. 10. Comparison between differences F03-P03 (F03: Fukushima 2003; P03: Capitaine et al. 2003) and the residuals of the "great inequality" term with respect to its approximation with a polynomial of degree 2 (in mas: $-4.4-6.8 t-0.7 t^{2}$ ): quantity $Q_{\mathrm{A}}$.

the short-period terms seem to have been correctly removed, the solution is significantly affected by a residual contribution of the GI terms. The HF04 solution of Harada \& Fukushima (2004), that has not been corrected for any long-period contributions, is obviously more seriously affected.

\subsection{Effect of the numerical ephemeris used as the reference}

In order to evaluate the effect of the numerical ephemeris that has been used for the fitting of the polynomials, comparisons between various such ephemerides have been made. Figures 11 and 12 illustrate the possible effect of the reference ephemeris (DE102, DE200, DE403, DE406) in the determination of $P_{\mathrm{A}}$ and $Q_{\mathrm{A}}$.

This illustration shows, in particular, that the choice of DE200 in the construction of VSOP87 did not play an important role in these comparisons. This moreover shows that any of the numerical ephemerides plotted in the Figures would have provided similar results for the P03 solution. Note that more extensive comparisons between VSOP planetary theories and JPL numerical ephemerides over 6000 years can be found in Chapront (2000).

Our basic time interval is 1600-2100, the overlap between DE200 and DE403, both of which are computed over a shorter

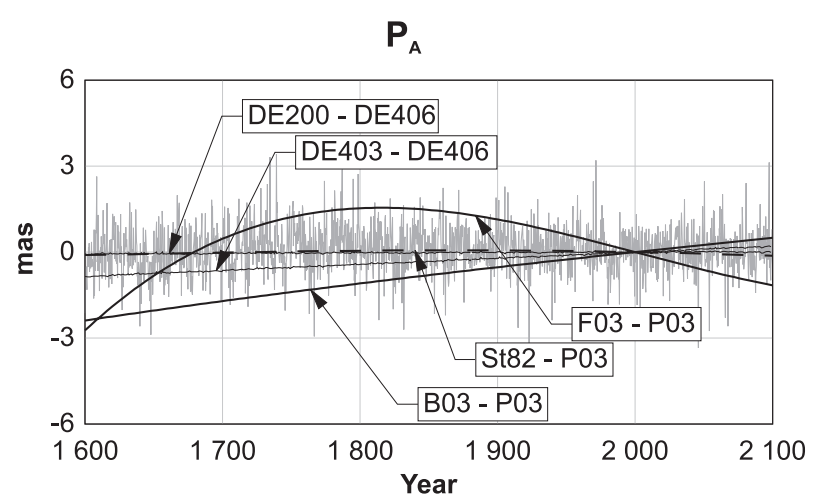

Fig. 11. Comparison of VSOP87 with various numerical ephemerides: quantity $P_{\mathrm{A}}$. The variations of the differences VSOP87*-DE406 (gray line) reveal the "noise" produced by the short-period terms in the analytical solutions. (B03: Bretagnon et al. 2003; F03: Fukushima 2003; P03: Capitaine et al. 2003; St82: Standish 1982).

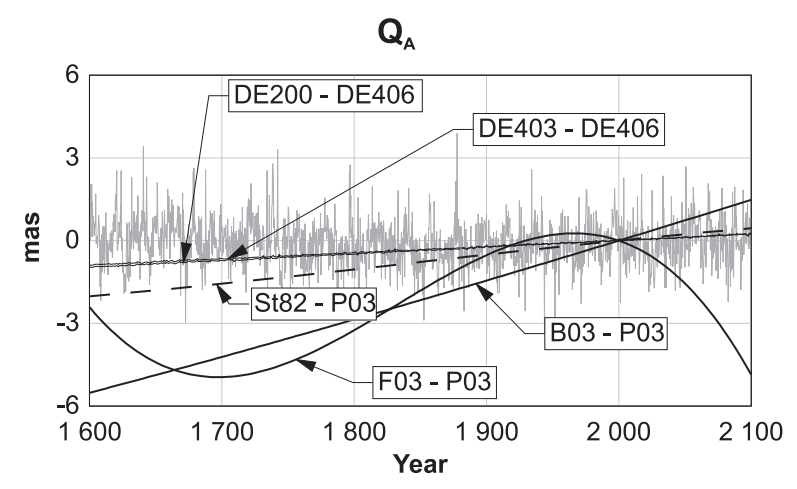

Fig. 12. Comparison of VSOP87 with various numerical ephemerides: quantity $Q_{\mathrm{A}}$. The variations of the differences VSOP87*-DE406 (gray line) reveal the "noise" produced by the short-period terms in the analytical solutions. (See legend of Fig. 11 for the precession models.)

interval compared with DE406. We obtained the following differences:

1) VSOP87* (adjusted polynomial) minus DE406 in gray;

2) DE200 minus DE406;

3) DE403 minus DE406;

4) B03 minus P03;

5) F03 minus P03;

6) St82 minus P03.

In these comparisons we bring together various numerical integrations or analytical solutions compared with DE406 (cases 1,2 and 3) and various secular ecliptic motions compared with P03 (cases 4, 5 and 6), taking P03 to represent the secular motion of the DE406 ecliptic variables. The shortperiod variations in 2) and 3) are eliminated in the differences. Note that, in order to perform the differencing, the numerical integrations have first been rotated into the ecliptic of VSOP87 with the appropriate angles $\phi$ and $\epsilon$ provided in Table 5 .

The above graphs show that the different JPL ephemerides provide close results for the corrections to $P_{\mathrm{A}}$ and $Q_{\mathrm{A}}$ (after an appropriate rotation to bring all the results into the same reference frame). In other words the distance between VSOP solutions with the original polynomials and with the adjusted 
Table 5. Rotation angles $\epsilon(\mathrm{DExxx}), \phi(\mathrm{DExxx})$ and secular trends between VSOP87 and various JPL numerical integrations DExxx (unit: milliarcsecond).

\begin{tabular}{lcccc}
\hline \hline Ephemeris & $\epsilon-23^{\circ} 26^{\prime} 21^{\prime \prime}$ & $\phi$ & $\left(\frac{\mathrm{d} P_{\mathrm{A}}}{\mathrm{d} t}\right)_{t=0}$ & $\left(\frac{\mathrm{d} Q_{\mathrm{A}}}{\mathrm{d} t}\right)_{t=0}$ \\
\hline DE102 & 408.56 & -93.66 & & \\
DE200 & 408.10 & -93.86 & -0.56 & -1.66 \\
DE403 & 408.56 & -53.68 & -0.35 & -1.68 \\
DE406 & 408.89 & -51.32 & -0.56 & -1.90 \\
\hline
\end{tabular}

polynomials are the same (or very close) irrespective of the choice of reference JPL ephemeris: DE200, DE403, DE406. This means that the evaluation of the inaccuracy in the secular variations of $p$ and $q$ in VSOP87 is only weakly dependent on the constants and parameters of the reference ephemeris.

When looking at the trends in the residuals between VSOP and DExxx, whatever the JPL reference ephemeris, the quantities $\left(\frac{\mathrm{d} P_{\mathrm{A}}}{\mathrm{d} t}\right)_{t=0}$ and $\left(\frac{\mathrm{d} Q_{\mathrm{A}}}{\mathrm{d} t}\right)_{t=0}$ show a systematic deviation, probably due to the analytical solution VSOP87, independently of the reference frame, the constants of integrations and other physical parameters of the JPL reference ephemeris. The deviations in $P_{\mathrm{A}}$ and $Q_{\mathrm{A}}$ are, in the case of DE406, of the order of $-0.6 \mathrm{mas} / \mathrm{cy}$ and $-1.9 \mathrm{mas} / \mathrm{cy}$, respectively. These trends can be seen in Figs. 11 and 12. The contributions due to the change of masses mentioned above are $-0.1 \mathrm{mas} / \mathrm{cy}$ and $-0.3 \mathrm{mas} / \mathrm{cy}$, respectively and are therefore much smaller. An illustration of the secular deviation between VSOP87 and DE406 is provided by Figs. 5 and 6 , the time interval covering 2 millennia. The thickness of the curves is evidence of the residuals due to shortperiod terms whose amplitudes are smaller than 3 mas. The "noise" produced by the short-period terms is the main limitation to the improvement of the secular variations described below.

\subsection{Tests of the inaccuracy in the secular terms of the analytical ephemeris}

The inaccuracy in the secular component of the analytical solution VSOP87 is clearly confirmed by the above comparison with various numerical ephemerides.

It is important to know whether the theoretical solution for the precession of the ecliptic would be improved by using a more recent analytical theory. For this purpose, the preliminary solution VSOP2000 (already mentioned in Sect. 2) has been tested, using, in particular, the secular variations of the ecliptic parameters $p$ and $q$ and a fit of the solution to DE403.

Whereas there is a significant reduction in the noise, coming from the improvement in the solution for the short-period terms, the conclusions mentioned above remain approximately the same for the secular component. Thus, it does not seem that this new analytical solution can bring real progress concerning the theoretical computation of the solution for the precession of the ecliptic. The same sort of differences appear when using DE406 as the reference.

The inaccuracy in the secular terms of the analytical solution can be due to (i) an insufficient degree of approximation of the analytical solution, and/or (ii) a less than perfect model for the effects due to the Moon (action of the Moon on the Earth-Moon barycenter), etc.

This shows the difficulty of computing the secular part of the EMB motion using an analytical method alone ${ }^{2}$ and justifies using a method that combines the strengths of an analytical and numerical representation. A comprehensive analytical representation of the periodic part of the motion allows the periodic effects to be eliminated (almost completely) from the fitting procedure, so that the polynomials representing the secular part take full advantage of the high accuracy of the numerical ephemerides.

This allows us to make a realistic estimate of the secular deficiency in the B03 solution for the precession of the ecliptic that is corrected when using the P03 solution.

\section{Precession of the equator}

\subsection{Comparison between the various solutions for the precession of the equator}

The various solutions for the developments for the precession quantities in longitude and obliquity (i.e. two basic quantities for the precession of the equator) are provided in Table 6 . This table clearly shows that whereas the difference between the IAU 2000 precession and the IAU 1976 precession is restricted to the corrections to the $t$ term, the recent solutions bring significant changes in the higher degree terms and increase the degree and resolution of the polynomials.

We also note that, except for the $t$ term, the Capitaine et al. (2003) solutions ( $\mathrm{P} 03$ and $\mathrm{P} 03_{\text {prel }}$ ) are the same at the microarcsecond level. We recall that this difference in the $t$ term is due to the correction applied to the final P03 solution for removing spurious effects arising from the procedure for providing the MHB estimates from observations (see Sect. 4.3 for more detail).

The quadratic difference in $\psi_{\mathrm{A}}$ of the $\mathrm{P} 03$ and F03 solutions with respect to the IAU 2000 model, and the large discrepancy in $\omega_{\mathrm{A}}$ with respect to F03 that has been noted in Figs. 1 and 2, clearly appear in Table 6.

As these solutions refer to various ecliptics and Earth models, different tests have been made to investigate the causes for the differences between the solutions: firstly the effect of the ecliptic used when solving the equations for the precession of the equator (Sect. 4.2) and secondly the influence of integration constants (Sect. 4.3) and the Earth model (Sect. 4.4). Then, the dynamical consistency of the solutions has been evaluated (Sect. 4.5) and the solutions have been checked against VLBI observations (Sect. 4.6). Note that there are also differences between contributions from geodesic precession, $p_{\mathrm{g}}$, (Brumberg 1991) in the different solutions, which are of the order of 1 mas in the $t$ term and below a few microarcseconds in the terms of higher degree. However, as this secular contribution is included in the integration constant of the solution, this does not need special study.

\footnotetext{
${ }^{2}$ However, further improvements of the VSOP analytical solution are likely in the foreseeable future (Simon 2004, private communication).
} 
Table 6. Comparisons between expressions for the precession of the equator (Sources: W94, Williams 1994; B03, Bretagnon et al. 2003; F03, Fukushima 2003; P03 and P03 prel, Capitaine et al. 2003); unit: milliarcsecond; the corresponding values of obliquity at epoch, $\epsilon_{0}$, are given in Table 8.

\begin{tabular}{|c|c|c|c|c|c|c|}
\hline Source & & $t$ & $t^{2}$ & $t^{3}$ & $t^{4}$ & $t^{5}$ \\
\hline IAU 1976 (L77) & & 5038778.4 & -1072.59 & -1.147 & & \\
\hline IAU 2000 & & 5038478.750 & -1072.59 & -1.147 & & \\
\hline W94 & & 5038456.501 & -1078.977 & -1.141 & 0.133 & \\
\hline B03 & & 5038478.750 & -1071.9530 & -1.14366 & 0.132832 & -0.0000940 \\
\hline F03 & $\psi_{\mathrm{A}}$ & 5038478.143 & -1079.1653 & -1.10654 & 0.129144 & \\
\hline P03 $3_{\text {prel }}$ & & 5038478.750 & -1079.0091 & -1.14044 & 0.132851 & -0.0000951 \\
\hline $\mathrm{P} 03$ & & 5038481.507 & -1079.0069 & -1.14045 & 0.132851 & -0.0000951 \\
\hline IAU 1976 (L77) & & 0.0 & 51.27 & -7.726 & & \\
\hline IAU 2000 & & -25.240 & 51.27 & -7.726 & & \\
\hline W94 & & -24.4 & 51.268 & -7.727 & 0.000000 & \\
\hline B03 & & -26.501 & 51.2769 & -7.72723 & -0.000492 & 0.0003329 \\
\hline F03 & $\omega_{\mathrm{A}}-\epsilon_{0}$ & -21.951 & 53.9411 & -7.19621 & +0.001907 & \\
\hline $\mathrm{P} 03_{\text {prel }}$ & & -25.240 & 51.2623 & -7.72502 & -0.000467 & 0.0003337 \\
\hline $\mathrm{P} 03$ & & -25.754 & 51.2623 & -7.72503 & -0.000467 & 0.0003337 \\
\hline
\end{tabular}

\subsection{Effect of the model of the ecliptic on the solution for the precession of the equator}

In order to evaluate the effect of the ecliptic on the precession solutions for the equator, we solved the same equations as for the $\mathrm{P} 03$ solution for the precession quantities $\psi_{\mathrm{A}}, \omega_{\mathrm{A}}, \epsilon_{\mathrm{A}}$ and $\chi_{\mathrm{A}}$ with the P03 values for the precession rates but using different solutions for the ecliptic:

- the IAU 1976 ecliptic;

- the B03 ecliptic (cf. W94);

- the F03 ecliptic.

The different solutions are set out in Table 7 together with the P03 solution.

Table 7 clearly shows that the milliarcsecond differences in the ecliptic solutions displayed in Table 1 are fully reflected in the coefficients for the $\epsilon_{\mathrm{A}}$ and $\chi_{\mathrm{A}}$ expressions, both of which refer directly to the ecliptic. On the other hand, the largest effect in the $\psi_{\mathrm{A}}$ and $\omega_{\mathrm{A}}$ expressions is of the order of 0.1 mas only. This proves that the large discrepancies:

(i) in $\omega_{\mathrm{A}}$ between the Fukushima (2003) solution (F03) and the other high precision solutions (of the order of 3 mas in the $t^{2}$ coefficient and 1 mas in the $t^{3}$ coefficient);

(ii) in $\psi_{\mathrm{A}}$ between the Bretagnon et al. (2003) solution (B03) and the other high precision solutions (of the order of 7 mas in the $t^{2}$ coefficient),

cannot be explained simply by the difference in the expressions for the ecliptic precession upon which the solutions for the equator are based.

\subsection{Effect of the integration constants on the solution for the precession of the equator}

a) Comparison between the integration constants of the various solutions

The recent precession models rely upon observed values for the precession rate in longitude and mean obliquity at epoch. The solutions differ (see Table 8) by a few milliarcseconds in the values for the obliquity at epoch and also the precession rates.

The difference between the values for the mean obliquity at epoch is due to different fits to observations (DE405 ephemerides or LLR observations).

Regarding the obliquity rate, the difference between the values is due to the fact that (i) the L77, B03 and W94 solutions correspond to theoretical computations without any observational constraint, the L77 and B03 solutions being for rigid Earth models and the W94 solution for a non-rigid Earth model, (ii) the F03 solution is derived from a fit to VLBI observations and (iii) the IAU 2000, P03 $3_{\text {prel }}$ and P03 solutions are based upon the MHB estimate, with some correction for the P03 solution (see below).

Regarding the precession in longitude, the difference between the values is due to the use of different estimates, the IAU 1976 one being determined by optical astronomy and the other ones by VLBI observations.

When solving the equations for the precession of the equator in the same way as for the P03 solutions, based on the P03 ecliptic, various solutions can be computed by changing the integration constants (i.e. the values for the precession rates in longitude and obliquity) and the Earth model.

Comparing the $\mathrm{P} 03$ and $\mathrm{P} 03_{\text {prel }}$ solutions shows that the effect of a 1 mas change in the integration constants is less than 
Table 7. Comparisons between P03-like precession solutions (P03 integration) based on different ecliptics (Sources: B03, Bretagnon et al. 2003; F03, Fukushima 2003; P03, Capitaine et al. 2003); unit: milliarcsecond; the corresponding values of obliquity at epoch, $\epsilon_{0}$, are given in Table 8 .

\begin{tabular}{|c|c|c|c|c|c|c|}
\hline Ecliptic & & $t$ & $t^{2}$ & $t^{3}$ & $t^{4}$ & $\overline{t^{5}}$ \\
\hline IAU 1976 & & 5038481.507 & -1079.097 & -1.147 & 0.131 & +0.0002 \\
\hline B03 & & 5038481.507 & -1078.973 & -1.140 & 0.133 & -0.0001 \\
\hline F03 & $\psi_{\mathrm{A}}$ & 5038481.507 & -1079.044 & -1.181 & 0.127 & -0.0001 \\
\hline $\mathrm{P} 03$ & & 5038481.507 & -1079.007 & -1.140 & 0.133 & -0.0001 \\
\hline IAU 1976 & & -25.754 & 51.244 & -7.722 & -0.0003 & 0.0003 \\
\hline B03 & & -25.754 & 51.269 & -7.725 & -0.0005 & 0.0003 \\
\hline F03 & $\omega_{\mathrm{A}}-\epsilon_{0}$ & -25.754 & 51.247 & -7.725 & -0.0001 & 0.0003 \\
\hline $\mathrm{P} 03$ & & -25.754 & 51.262 & -7.725 & -0.0005 & 0.0003 \\
\hline IAU 1976 & & -46840.754 & -0.603 & 1.816 & +0.029 & -0.0000 \\
\hline B03 & & -46835.304 & -0.176 & 2.002 & -0.001 & -0.0002 \\
\hline F03 & $\epsilon_{\mathrm{A}}-\epsilon_{0}$ & -46838.403 & -2.864 & 1.471 & -0.002 & +0.0009 \\
\hline $\mathrm{P} 03$ & & -46836.769 & -0.183 & 2.003 & -0.001 & -0.0000 \\
\hline IAU 1976 & & +10552.642 & -2380.460 & -1.122 & 0.160 & -0.0001 \\
\hline B03 & & +10557.685 & -2381.378 & -1.209 & 0.170 & -0.0001 \\
\hline F03 & $\chi_{\mathrm{A}}$ & +10553.205 & -2381.554 & -1.064 & 0.141 & -0.0002 \\
\hline $\mathrm{P} 03$ & & +10556.403 & -2381.429 & -1.212 & 0.171 & -0.0001 \\
\hline
\end{tabular}

Table 8. Comparisons between obliquity and precession rate values of the various solutions (see description of the labels in the caption of Table 6); (unit: arcsecond).

\begin{tabular}{llll}
\hline \hline Source & $\epsilon_{0}$ & $\psi_{1}$ & $\psi_{1} \sin \epsilon_{0}$ \\
\hline IAU 1976 & 84381.448 & 5038.7784 & 2004.310941 \\
IAU 2000 & 84381.448 & 5038.47875 & 2004.191747 \\
W94 & 84381.409 & 5038.456501 & 2004.182023 \\
B03 & 84381.4088 & 5038.478750 & 2004.190869 \\
F03 & 84381.4062 & 5038.478143 & 2004.190569 \\
P03 & 84381.406 & 5038.478750 & 2004.190806 \\
P03 & 84381.406 & 5038.481507 & 2004.191903 \\
\hline
\end{tabular}

a few $\mu$ as in the higher degree terms of the precession expressions. The 3 mas difference of the F03 value for the precession rate in obliquity with respect to the IAU 2000 value cannot therefore explain the milliarcsecond discrepancy of the $t^{2}$ and $t^{3}$ coefficients in the expression for $\omega_{\mathrm{A}}$ between the F03 solutions and the other solutions.

We can also conclude that next revision of the values for the precession rates, expected to less than 1 mas, will change the coefficients of the precession expressions for the equator by only a few microarcseconds.

b) Dependence of the VLBI estimate for the precession rate on the obliquity of the ecliptic

It is important to note that the value for the precession rate corresponding to a given ecliptic has to be consistent with the quantity that the observations actually provided, depending on which parameter they are actually sensitive to. When using observations that are not sensitive to an ecliptic, as is the case for VLBI, the estimate of the correction to the precession rate in longitude, $\psi_{\mathrm{A}}$, depends, through the precession model that is used, on the ecliptic to which it is referred. The dependence effect is shown in Fig. 13. VLBI not being sensitive to an ecliptic, the VLBI-estimated correction to the precession rate in longitude is in fact associated with the value $\epsilon_{01}$ for the obliquity of the ecliptic at $\mathbf{J} 2000$ that was used in the VLBI reductions (namely the IAU 1976 value) for providing the celestial pole offsets, the actual "estimated quantity" being $\psi_{\mathrm{A}} \sin \epsilon_{0}$. Thus, if the obliquity at epoch is changed from $\epsilon_{01}$ (corresponding to ecliptic 1) to $\epsilon_{02}$ (corresponding to ecliptic 2) when adopting a new precession model, then the correction to the precession rate to be used in the new model has to be changed by $\psi_{\mathrm{A} 2}-\psi_{\mathrm{A} 1}$ in order to keep unchanged the quantity $\psi_{\mathrm{A}} \sin \epsilon_{0}$ to which VLBI is actually sensitive. If the estimated value for $\psi_{\mathrm{A}}$ is kept unchanged when the obliquity at epoch is changed from $\epsilon_{01}$ to $\epsilon_{02}$, then the quantity $\psi_{\mathrm{A}} \sin \epsilon_{0}$ is changed by $\psi_{\mathrm{A}}\left[\sin \epsilon_{02}-\sin \epsilon_{01}\right]$ (i.e. $5038^{\prime \prime} .47875\left[\sin \left(8438123^{\prime \prime}\right.\right.$ '406) $\left.\sin \left(8438123^{\prime \prime} .448\right)\right] \sim 1$ mas and the corresponding value for the precession rate is therefore in error by 2.37 mas.

This effect was taken into account in the P03 solution. Note that additional spurious effects in the precession rates in longitude and obliquity were also considered when computing the $\mathrm{P} 03$ solution. These latter effects come from the pre-2003 VLBI procedure for estimating the celestial pole offsets; this treated the biases as if they were nutation components, applying them at date rather than at epoch, and omitting the equinox offset, consequently introducing spurious coupling terms of $-384 \mu \mathrm{as} / \mathrm{cy}$ in longitude and $+514 \mu \mathrm{as} / \mathrm{cy}$ in obliquity (Capitaine et al. 2003). 


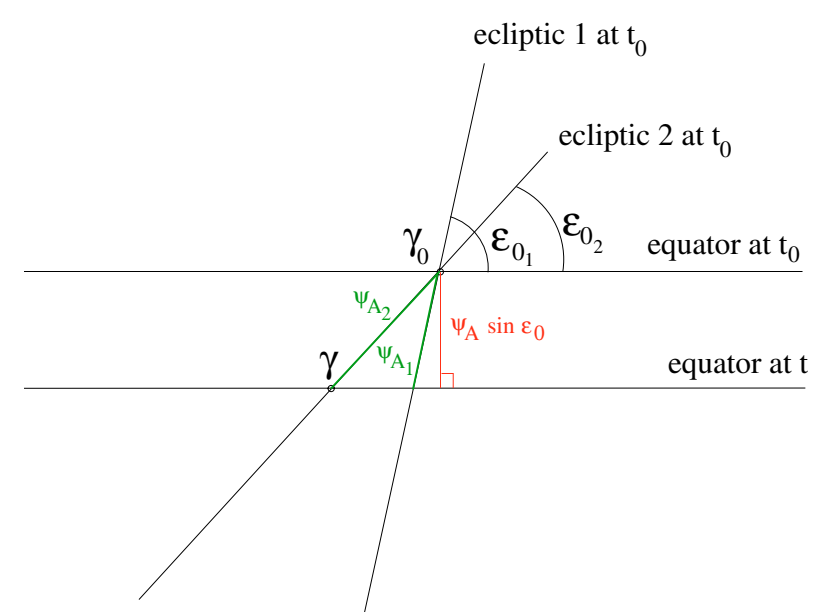

Fig. 13. Dependence of the VLBI estimate for the precession rate in longitude, $\psi_{\mathrm{A}}$, on the obliquity of the ecliptic ( 1 or 2 ) to which it is referred.

\subsection{Effect of the Earth model on the solution for the precession of the equator}

In order to identify the effects of the Earth model in the solutions, we used successively, based on the IAU 2000 integration constants:

- a purely rigid-Earth model (denoted RE);

- a non-rigid Earth model (denoted NREt) by adding to the RE model the tidal contributions both to the obliquity rate and precession rate;

- a non-rigid Earth model (denoted NREtJ2d) by adding to the model NREt the $J_{2}$ rate effect with the same amplitude as for the $\mathrm{P} 03$ solution;

- the P03 $3_{\text {prel }}$ solution, which is based on the IAU 2000 precession rates and corresponds to a non-rigid Earth model including the tidal and $J_{2}$ rate contributions to precession and the MHB non-linear contribution as well (see Mathews et al. 2002 or Capitaine et al. 2003 for more detail).

Table 9 provides a comparison between solutions corresponding to the various Earth models up to the 3rd degree (the effect being entirely negligible in the coefficients of higher degrees).

The changes in the solution according to the Earth model clearly appear in Table 9, which in particular shows that the largest changes are of the order of 2.4 mas in the secular term of the expressions for $\omega_{\mathrm{A}}$ and $\epsilon_{\mathrm{A}}$, due to the tidal contribution and 7.0 mas in the quadratic term in longitude, due to the $J_{2}$ rate effect. The only other non-negligible Earth model effect is the tidal contribution of $120 \mu$ as in the quadratic term in longitude, all the other coefficients being insensitive to the Earth model at a microarcsecond level.

Regarding the largest Earth model effects, note that (i) the tidal contribution in obliquity rate is automatically included in the estimated value from VLBI; (ii) the $J_{2}$ rate value that causes the largest change in the precession model for the equator is the most significant limitation of the model. An accurate representation of the $J_{2}$ time variations would need the use of spacegeodetic determination of the time variations in the geopotential on a regular basis (see Bourda \& Capitaine 2004).
Table 9. Comparisons between "P03-like" precession solutions (P03 integration constants and P03 ecliptic) for the equator based on different Earth models (unit: milliarcsecond); $\epsilon_{0}=84381.406$ arcsec.

\begin{tabular}{|c|c|c|c|c|}
\hline Source & & $t$ & $t^{2}$ & $t^{3}$ \\
\hline RE & & 5038478.750 & -1071.880 & -1.143 \\
\hline NREt & $\psi_{\mathrm{A}}$ & 5038478.750 & -1072.010 & -1.142 \\
\hline NREtJ2d & & 5038478.750 & -1079.010 & -1.140 \\
\hline $\mathrm{P} 03_{\text {prel }}$ & & 5038478.750 & -1079.009 & -1.140 \\
\hline $\mathrm{RE}$ & & -27.640 & 51.263 & -7.725 \\
\hline NREt & $\omega_{\mathrm{A}}-\epsilon_{0}$ & -25.240 & 51.262 & -7.725 \\
\hline NREtJ2d & & -25.240 & 51.262 & -7.725 \\
\hline $\mathrm{P} 03_{\text {prel }}$ & & -25.240 & 51.262 & -7.725 \\
\hline $\mathrm{RE}$ & & -46838.655 & -0.183 & 2.003 \\
\hline NREt & $\epsilon_{\mathrm{A}}-\epsilon_{0}$ & -46836.255 & -0.183 & 2.003 \\
\hline NREtJ2d & & -46836.255 & -0.183 & 2.003 \\
\hline $\mathrm{P} 03_{\text {prel }}$ & & -46836.255 & -0.183 & 2.003 \\
\hline $\mathrm{RE}$ & & +10556.403 & -2381.427 & -1.216 \\
\hline NREt & $\chi_{\mathrm{A}}$ & +10556.403 & -2381.428 & -1.216 \\
\hline NREtJ2d & & +10556.403 & -2381.428 & -1.212 \\
\hline $\mathrm{P} 03_{\text {prel }}$ & & +10556.403 & -2381.429 & -1.212 \\
\hline
\end{tabular}

This clearly shows that the difference between the B03 solution and the other solutions both for the obliquity rate and the quadratic term in the precession in longitude can be explained by the fact that, except for the secular term in longitude, the B03 solution is (as the L77 solution) relative to a rigid Earth, whereas the other solutions refer to non-rigid-Earth models.

This is confirmed by a similar comparison (Table 10), based on the same Earth models as above, but using the Bretagnon et al. 2003 integration constants and ecliptic (B03). Note that such comparisons revealed that the B03 solution, that is known to correspond to a rigid Earth, in fact appears to include the $120 \mu \mathrm{as} / \mathrm{cy}^{2}$ tidal contribution in longitude (see the difference in the B03 solution between Tables 10 and 11), but not the corresponding secular contribution in obliquity.

\subsection{Test of the dynamical consistency of the solutions}

Given a model for the precession of the ecliptic, integration constants for the precession rates and an Earth model, solving the equations for the precession of the equator provides solutions that are "dynamically consistent". We have tested this aspect of the various published models by a) solving the same equations as were used to obtain the P03 solutions but using the models (ecliptic, integration constants, Earth model, etc.) as close as possible to those of the IAU 1976, W94, B03 and F03 solutions (i.e. Table 1 for the ecliptic and Table 8 for the integration constants) and b) comparing the numerical values of the $t^{2}$ coefficients of the solutions with the theoretical values derived from the analytical expression as provided in Table 7 of Capitaine et al. (2003). We also tested the dynamical consistency of the IAU 2000 solution, which differs from the 
Table 10. Comparisons between "B03-like" precession solutions (i.e. Bretagnon et al. 2003 integration constants and ecliptic (B03)) for the equator based on different Earth models (unit: milliarcsecond); $\epsilon_{0}=84381.4088$ arcsec.

\begin{tabular}{lrrrr}
\hline \hline Source & & \multicolumn{1}{c}{$t$} & $t^{2}$ & $t^{3}$ \\
\hline RE & & 5038478.750 & -1071.852 & -1.142 \\
NREt & $\psi_{\mathrm{A}}$ & 5038478.750 & -1071.983 & -1.142 \\
NREtJ2d & & 5038478.750 & -1078.983 & -1.140 \\
cf. P03 & & 5038478.750 & -1078.983 & -1.140 \\
\hline RE & & -26.501 & 51.269 & -7.725 \\
NREt & $\omega_{\mathrm{A}}-\epsilon_{0}$ & -24.101 & 51.269 & -7.725 \\
NREtJ2d & & -24.101 & 51.269 & -7.725 \\
cf. P03 & & -24.101 & 51.269 & -7.725 \\
\hline RE & & -46836.051 & -0.175 & 2.002 \\
NREt & & -46833.651 & -0.175 & 2.002 \\
NREtJ2d & & -46833.651 & -0.175 & 2.002 \\
cf. P03 & & -46833.651 & -0.175 & 2.002 \\
\hline RE & & +10557.685 & -2381.377 & -1.213 \\
NREt & $\chi_{\mathrm{A}}$ & +10557.685 & -2381.377 & -1.213 \\
NREtJ2d & & +10557.685 & -2381.377 & -1.209 \\
cf. P03 & & +10557.685 & -2381.377 & -1.209 \\
\hline & & & &
\end{tabular}

IAU 1976 solution only by corrections to the precession rates in longitude and obliquity.

\section{a) Integration of the equations}

Solutions corresponding to the models and integration constants of various solutions are provided in Table 11, and comparisons with the original solutions are shown in Table 12. Note that the Earth model corresponding to the original solutions is known, except for the F03 and IAU 2000 solutions for which we tested the various models described in Sect. 4.4 and used the one that showed the best agreement. Table 12 shows very good agreement, with the exception of the F03 solution. The differences with respect to the L77, W94 and B03 solutions are of the order of a few tens of microarcseconds (except for terms of degree higher than 3 for the L77 solution which were not provided in the original solution), which reflects their dynamical consistency. For the F03 solution, on the other hand, the differences reach several milliarcseconds in obliquity; the differences resulting from the Earth model being generally much lower than this (cf. Table 9), the F03 solution therefore appears not to be dynamically consistent.

The differences of the IAU 2000 precession variables with respect to a solution that is obtained by solving the precession equations based on the IAU 2000 precession rates as integration constants, the IAU 1976 ecliptic and an Earth model with a tidal contribution only (NREt) reach 0.5 mas in the quadratic term of $\psi_{\mathrm{A}}$. Note that the differences corresponding to the $\mathrm{P} 03_{\text {prel }}$ and $\mathrm{P} 03$ solutions would obviously be zero as these solutions have been obtained by solving the P03 dynamical equations of precession. b) Analytical coefficients

Theoretical expressions for the precession quantities as derived by an analytical solution of the equations for the precession of the equator can be used for checking the dynamical consistency of the solutions. Expressions for the basic precession quantities were provided in Table 7 of Capitaine et al. (2003) as functions of the coefficients $\left(r_{i}\right)_{i=0,2}$ and $\left(s_{i}\right)_{i=0,2}$ of the polynomial developments for the precession rates $r_{\psi}$ in ecliptic longitude and $r_{\epsilon}$ in obliquity, with respect to inertial space and $\left(c_{i}\right)_{i=1,3}$ and $\left(s_{i}\right)_{i=1,3}$ for the ecliptic quantities $P_{\mathrm{A}}$ and $Q_{\mathrm{A}}$. Comparing the $t^{2}$ coefficients of the solutions to their theoretical values provided by the expressions as function of the largest components of the precession rates $P_{0}$ and $p_{\mathrm{g}}$ in longitude and $u_{0_{1}}$ in obliquity (cf. Table 13) provides a relevant test of dynamical consistency.

The comparisons confirm that all the recent solutions are dynamically consistent, with the exception of the F03 solutions for the precession of the equator.

\subsection{Test of the solutions against VLBI observations}

VLBI observations provide on a regular basis the "celestial pole offsets" that represent the residuals of the actual position of the CIP in the GCRS with respect to that provided by the precession-nutation model plus the frame biases. Time series of VLBI celestial pole offsets are therefore potentially a powerful and conclusive way of discriminating between different models for the precession-nutation of the equator.

This is indeed the case for nutation, but, in contrast, due to the insufficient length of the available VLBI series ( $<20$ years), tests of the precession solutions against VLBI data do not at this stage allow useful conclusions to be reached regarding how well the different models agree with the observations. We used both the IAU 2000A model and the P03 model to predict the CIP $X, Y$ and compared the results with VLBI data spanning 1985.0-2003.9; the two models gave very similar results, any differences between them being lost in the noise (overall about $400 \mu$ as rms in each of $X$ and $Y$ though improving year by year). This result suggests that the VLBI data are unable at present to discriminate between any of the various models for the precession of the equator. A more positive conclusion can of course be drawn from these tests, namely that the existing implementations of the IAU 2000A models provide an effective practical tool for predicting the path of the celestial pole, in spite of the known shortcomings from a theoretical point of view.

\section{Summary}

In this paper we have compared the most recent precession solutions (Bretagnon et al. 2003; Fukushima 2003; Capitaine et al. 2003), respectively denoted B03, F03 and P03, that provide high-precision precession expressions consistent with the IAU 2000A precession-nutation model (Mathews et al. 2002) and are potential replacements for the precession component of IAU 2000A, offering improved dynamical consistency and providing a better basis for subsequent improvements in the 
Table 11. Solutions denoted as (*)-like corresponding to the models, integration constants and ecliptic precession of various precession solutions (Sources: W94, Williams 1994; B03, Bretagnon et al. 2003; F03, Fukushima 2003),(unit: milliarcsecond); the corresponding values of obliquity at epoch, $\epsilon_{0}$, are given in Table 8 .

\begin{tabular}{|c|c|c|c|c|c|c|}
\hline Source & & $\bar{t}$ & $t^{2}$ & $t^{3}$ & $\overline{t^{4}}$ & $\overline{t^{5}}$ \\
\hline (IAU 1976)-like & & 5038778.400 & -1072.590 & -1.147 & 0.131 & -0.0001 \\
\hline (IAU 2000)-like & & 5038478.750 & -1072.102 & -1.149 & 0.131 & +0.0002 \\
\hline (W94)-like & & 5038456.501 & -1078.977 & -1.141 & 0.133 & -0.0001 \\
\hline (B03)-like & $\psi_{\mathrm{A}}$ & 5038478.750 & -1071.970 & -1.142 & 0.133 & -0.0001 \\
\hline (F03)-like & & 5038478.143 & -1079.047 & -1.181 & 0.127 & -0.0001 \\
\hline (IAU 1976)-like & & 0.0 & 51.271 & -7.726 & -0.0003 & 0.0000 \\
\hline (IAU 2000)-like & & -25.240 & 51.244 & -7.724 & -0.0003 & 0.0003 \\
\hline (W94)-like & & -24.400 & 51.268 & -7.727 & -0.0005 & 0.0003 \\
\hline (B03)-like & $\omega_{\mathrm{A}}-\epsilon_{0}$ & -26.501 & 51.269 & -7.725 & -0.0005 & 0.0003 \\
\hline (F03)-like & & -21.951 & 51.247 & -7.725 & -0.0001 & 0.0003 \\
\hline (IAU 1976)-like & & -46815.000 & -0.582 & 1.813 & 0.029 & -0.0000 \\
\hline (IAU 2000)-like & & -46840.240 & -0.603 & 1.814 & 0.029 & -0.0000 \\
\hline (W94)-like & & -46833.960 & -0.174 & 2.000 & -0.001 & -0.0001 \\
\hline (B03)-like & $\epsilon_{\mathrm{A}}-\epsilon_{0}$ & -46836.051 & -0.175 & 2.002 & -0.001 & -0.0002 \\
\hline (F03)-like & & -46834.600 & -2.864 & 1.471 & -0.002 & +0.0010 \\
\hline (IAU 1976)-like & & +10552.608 & -2380.644 & -1.125 & +0.161 & -0.0001 \\
\hline (IAU 2000)-like & & +10552.642 & -2380.459 & -1.126 & +0.159 & -0.0001 \\
\hline (W94)-like & & +10557.700 & -2381.366 & -1.209 & +0.170 & -0.0002 \\
\hline (B03)-like & $\chi_{\mathrm{A}}$ & +10557.685 & -2381.377 & -1.213 & +0.170 & -0.0001 \\
\hline (F03)-like & & +10553.205 & -2381.553 & -1.064 & -0.141 & -0.0002 \\
\hline
\end{tabular}

Table 12. Differences of the solutions derived from a P03-like integration (Capitaine et al. 2003) and based on the ecliptic (Table 1) and integration constants (Table 8) of various solutions (and Earth models when known) with respect to the original solutions (Table 6) (unit: microarcsecond).

\begin{tabular}{|c|c|c|c|c|c|c|}
\hline Source & & $t$ & $\overline{\overline{t^{2}}}$ & $\overline{t^{3}}$ & $\overline{t^{4}}$ & $\overline{t^{5}}$ \\
\hline diff wrt IAU 1976 & & 0 & 0 & 0 & 131 & -1 \\
\hline diff wrt IAU 2000 & & 0 & 488 & 2 & 131 & 0 \\
\hline diff wrt W94 & & 0 & 0 & 0 & 0 & 0 \\
\hline diff wrt B03 & $\psi_{\mathrm{A}}$ & 0 & -17 & 1 & 0 & 0 \\
\hline diff wrt F03 & & 0 & 101 & 74 & -2 & 0 \\
\hline diff wrt IAU 1976 & & 0 & -1 & 0 & 0 & 0 \\
\hline diff wrt IAU 2000 & & 0 & -26 & 2 & 0 & 0 \\
\hline diff wrt W94 & & 0 & 0 & 0 & -1 & 0 \\
\hline diff wrt B03 & $\omega_{\mathrm{A}}$ & 0 & -8 & 2 & 0 & 0 \\
\hline diff wrt F03 & & 0 & -2694 & -529 & 10 & 0 \\
\hline diff wrt IAU 1976 & & 0 & 0 & 8 & 29 & 0 \\
\hline diff wrt IAU 2000 & & 0 & -13 & 1 & 29 & 0 \\
\hline diff wrt W94 & & 0 & 0 & 0 & 0 & 0 \\
\hline diff wrt B03 & $\epsilon_{\mathrm{A}}$ & 0 & -8 & 2 & 0 & 0 \\
\hline diff wrt F03 & & 0 & -2694 & -529 & -2 & 1 \\
\hline diff wrt IAU 1976 & & 8 & 4 & 0 & 161 & 0 \\
\hline diff wrt IAU 2000 & & 42 & 181 & -1 & 159 & -1 \\
\hline diff wrt W94 & & 0 & 0 & -1 & 0 & 0 \\
\hline diff wrt B03 & $\chi_{\mathrm{A}}$ & -1 & 0 & 0 & 0 & 0 \\
\hline diff wrt F03 & & 0 & 0 & 0 & 0 & 0 \\
\hline
\end{tabular}

Table 13. Expressions for the two first coefficients of the polynomial classical precession quantities.

\begin{tabular}{lcc}
\hline \hline Quantity & Coefficient of $t$ & Coefficient of $t^{2}$ \\
\hline$\psi_{\mathrm{A}}$ & $r_{0}$ & $\frac{1}{2}\left(r_{1}+r_{0} c_{1} \cot \epsilon_{0}-\frac{u_{0} s_{1}}{\sin ^{2} \epsilon_{0}}\right)$ \\
\hline$\omega_{\mathrm{A}}$ & $u_{0}$ & $\frac{1}{2}\left(u_{1}+r_{0} s_{1}\right)$ \\
\hline$\epsilon_{\mathrm{A}}$ & $c_{1}+u_{0}$ & $c_{2}+\frac{1}{2}\left(u_{1}-r_{0} s_{1}+s_{1}^{2} \cot \epsilon_{0}\right)$ \\
\hline$\chi_{\mathrm{A}} \sin \epsilon_{0}$ & $s_{1}$ & $s_{2}+r_{0} c_{1}-s_{1} \cot \epsilon_{0}\left(u_{0}+c_{1}\right)$ \\
\hline
\end{tabular}

conventions. We compared the expressions for the basic parameters of the above solutions both for the ecliptic and the equator and investigated the possible physical and computational reasons for their differences. This provided estimated deficiencies in the solutions and leads to a realistic evaluation of their respective accuracies. We also considered the latest solution for planetary precession by Harada \& Fukushima (2004), denoted HF04.

The sizes of the changes in the terms of the various solutions with respect to IAU 2000 are summarized in Fig. 14.

Regarding the precession of the ecliptic, we evaluated the accuracy of the solutions by comparing them to numerical ephemerides (Sects. 3.2 and 3.5) and we investigated some possible causes for the differences. In particular, we evaluated the effect of the long-period variations of the ecliptic in the 


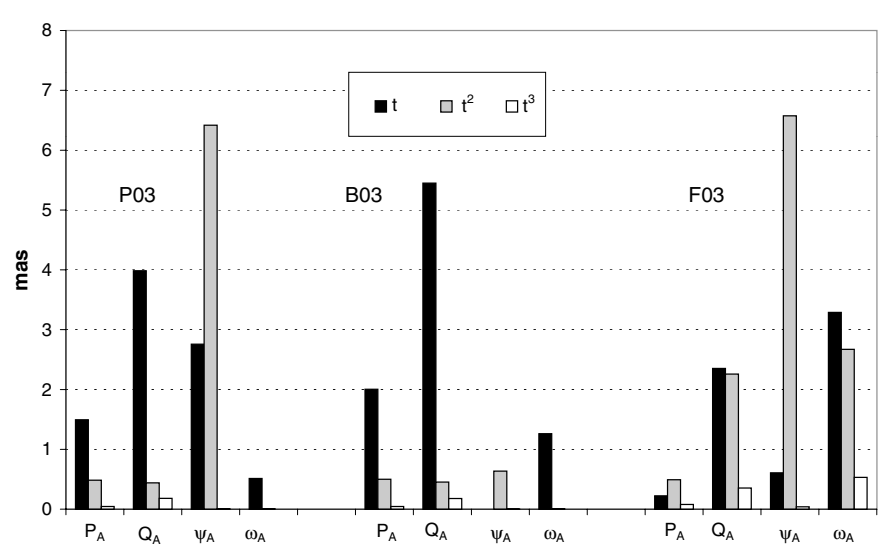

Fig. 14. The size of the changes in the terms of the various solutions with respect to IAU 2000 (P03: Capitaine et al. 2003; B03: Bretagnon et al. 2003; F03: Fukushima 2003).

determination of the polynomial part (Sect. 3.3) that appeared as crucial in the case of the F03 and HF04 solutions. We also evaluated the effect of the numerical ephemerides that were used for the fit of the polynomial (Sect. 3.4) that was crucial in the case of the B03 and P03 solutions. We concluded that:

- the current IAU expressions for the precession of the ecliptic need to be improved, both by revising the numerical values of the coefficients and by extending the numerical resolution and degree of the current developments;

- the inaccuracies in the current IAU expressions are evaluated as being of the following order:

. a few milliarcseconds in the secular terms;

- a few hundred microarcseconds in the quadratic terms for $P_{\mathrm{A}}$ and $Q_{\mathrm{A}}$ and cubic term for $Q_{\mathrm{A}}$;

- a few tens of microarcseconds in the cubic term for $P_{\mathrm{A}}$; . one microarcsecond or less in the $t^{4}$ and $t^{5}$ terms for both variables;

- improved models for the precession of the ecliptic are available that provide developments up to the fifth degree with microarcsecond numerical precision after ten centuries;

- among the available solutions, the solutions W94, B03 and P03 are all consistent except for slope differences of a few mas/cy;

- the Fukushima (2003) solution (F03) and Harada \& Fukushima (2004) solutions (HF04) are largely affected by the residual contribution of the long periodic terms that have been incorrectly corrected:

. the discrepancies appearing in the F03 solution can be explained by the method used for the fit to DE405, due to (i) the insufficient length of the interval for the fit (600 years) and (ii) the imperfect method to discriminate between the secular motion of the ecliptic and very-long periodic components as the GI term;

the much larger discrepancies appearing in the HF04 solution can be explained by the method used for the fit to DE405 over the 600-yr interval that was modified with respect to F03 in the sense that it totally ignored the contribution of the terms of very long periods;

- the discrepancy in the F03 and HF04 solutions is confirmed by a comparison with an independent fit to DE102 by Standish (1982) that was based on a method similar to F03, but over a longer interval and a correct removal of the GI term;

- there is an inaccuracy of a few milliarcseconds in the secular term of the current analytical ephemerides for the EMB motion that was not fully taken into account through the corresponding theoretical computation;

- this inaccuracy results in a secular deficiency in the Williams (1994) (W94) and Bretagnon et al. (2003) (B03) solutions that was corrected in the Capitaine et al. (2003) solution (P03) through a fit to DE406, the accuracy of which has been evaluated as being of the order of $0.05 \mathrm{mas} / \mathrm{cy}$ in the interval 1000-3000.

Regarding the precession of the equator, we evaluated the effect of the ecliptic used when solving the equations for the precession of the equator (Sect. 4.2) and the influence of the Earth model (Sect. 4.4) and integration constants (Sect. 4.3). We moreover evaluated the dynamical consistency of the solutions (Sect. 4.5) and tested them against VLBI observations (Sect. 4.6). We concluded that:

- the IAU 2000 expressions for the precession of the equator would need to be improved by a better numerical resolution of the coefficients and by extending the degree of the developments;

- the quadratic and cubic coefficients of the IAU 2000 precession in obliquity are accurate at a $10 \mu$ as level;

- the quadratic coefficient of the IAU 2000 precession in longitude may be in error by about 7 mas, whereas the cubic coefficient is accurate at a $10 \mu$ as level;

- the accuracy of the expression for the precession in longitude is strongly limited by the uncertainty in the model for the $J_{2}$ time variations;

- the F03 solution (Fukushima 2003) is not dynamically consistent;

- among the solutions that are dynamically consistent, only the P03 solution (Capitaine et al. 2003) takes into account a realistic Earth model and integration constants;

- the precession rate in longitude to be used in the precession model for the equator is dependent on the obliquity of the ecliptic model;

- a new precession model for the equator should be associated with revised determination of the values for the precession rates based on the most recent geophysical models and VLBI observations;

- VLBI records are at this stage unable to discriminate between the different solutions.

An IAU Division I Working Group on "precession and the ecliptic" was created at the XXVth General Assembly in order to address the issue of developing new models, and began work in September 2003; it is hoped that the present paper will assist this work. In the opinion of the authors, the IAU 2000 precession of the ecliptic (i.e. the IAU 1976 model) needs to be improved upon, and the present studies have looked at various solutions that agree to a few mas per century and are candidates for such a role. These studies have identified expressions for the ecliptic precession quantities that are accurate to about 0.05 mas/cy over a two-millennium interval centered on J2000. 
Table A.1. Notations and corresponding references for specific models used in this paper.

\begin{tabular}{lll}
\hline \hline Notation & Authors & Reference \\
\hline B03 & Bretagnon et al. (2003) & A\&A, 400, 785 \\
F03 & Fukushima (2003) & AJ, 126, 494 \\
HF04 & Harada \& Fukushima (2004) & AJ, 127, \\
L77 & Lieske et al. (1977) & A\&A, 58, 1 \\
MHB & Mathews et al. (2002) & JGR, 107, B4 \\
P03 & Capitaine et al. (2003) & A\&A, 412, 567 \\
St82 & Standish (1982) & A\&A, 114, 297 \\
W94 & Williams (1994) & AJ, 108, 711 \\
\hline
\end{tabular}

On the other hand, replacing the IAU 2000 precession of the equator remains an open question: improvements are still needed both in the models (mainly for the $J_{2}$ rate) and the fit to observations (for the integration constants) before adopting a new model that would represent a significant improvement with respect to IAU 2000.

The present studies have provided a theoretical and experimental basis for such future improvements, including in P03 a set of procedures for generating new precession models, given improved observational inputs.

Acknowledgements. We thank the referee and the editor of this paper for their suggestions for improving the presentation of the text.

\section{Appendix A: Notations for specific models}

Table A.1 provides the references corresponding to the notations used in this paper for designating specific precession models.

\section{Appendix B: Note added to the paper after acceptance}

Since this work was carried out, the authors have looked further at the VLBI procedures that lay behind the IAU 2000 precession rates. This reexamination has led them to change their interpretation of the spurious effects of the non-rigorous pre-2003 VLBI procedure in the MHB estimated precession rates (see penultimate line in Sect. 4.3) requiring that the corrections for these effects should in fact be applied to the MHB values with the opposite sign than that proposed in Paper P03 (Capitaine et al. 2003). Consequently, the P03 solution for the precession of the equator, although it is dynamically consistent and has the qualities that are reported in this paper in comparison with respect to other solutions, needs a small correction to its integration constants (cf. Sect. 6.2.4 of the P03 paper). This change will provide a solution for the precession of the equator which will best agree with the IAU 2000 precession rates values freed from the actual effects of the non-rigorous pre-2003 VLBI procedure.

The change in the integration constants with respect to $\mathrm{P} 03$ corresponds to revised secular terms of 5038.' 480732 in longitude and $-0{ }^{\prime}$.024726 in obliquity. Note that fits to VLBI data confirmed that such a change in the secular terms of the precession model for the equator is within the present uncertainty of VLBI observations, and so the revised solution is not superior to $\mathrm{P} 03$ in a practical sense. It should also be noted that such a change (i) does not affect the P03 solution for the ecliptic (i.e. expressions for $P_{\mathrm{A}}, Q_{\mathrm{A}}, \pi_{\mathrm{A}}, \Pi_{\mathrm{A}}$ remain unchanged), and (ii) does not induce changes in the revised solution for the precession of the equator larger than one microarcsecond in the higher degree terms of the expressions, except for a change of the order of $10 \mu$ as in the quadratic term in longitude and related quantities.

\section{References}

Bidart, P. 2000, Thèse, Observatoire de Paris

Bourda, G., \& Capitaine, N. 2004, in preparation

Bretagnon, P., \& Francou, G. 1988, A\&A, 202, 309

Bretagnon, P., Rocher, P., \& Simon, J.-L. 1997, A\&A, 319, 305

Bretagnon, P., Fienga, A., \& Simon, J.-L. 2003, A\&A, 400, 785

Brumberg, V. 1991, Essential Relativistic Celestial Mechanics (Adam Hilger Pub)

Capitaine, N., Wallace, P. T., \& Chapront, J. 2003, A\&A, 412, 567

Chapront, J., Chapront-Touzé, M., \& Francou, G. 2002, A\&A, 387, 700

Chapront, J. 2000, Celest. Mech. Dyn. Astr., 78, 7582

Fukushima, T. 2003, AJ, 126, 494

Harada, W., \& Fukushima, T. 2004, AJ, 127, 531

IERS Standards 1992, IERS Technical Note, ed. D. D. McCarthy (Observatoire de Paris)

IERS Conventions 2003, IERS Technical Note 32, ed. D. D. McCarthy, \& G. Petit, in press

Lieske, J. H., Lederle, T., Fricke, W., \& Morando, B. 1977, A\&A, 58, 1

Mathews, P. M., Herring, T. A., \& Buffett, B. A. 2002, J. Geophys. Res., 107, B4, 10.1029/2001JB000165

Moisson, X., \& Bretagnon, P. 2001, VSOP 2000, Celest. Mech. Dyn. Astr., 80, 205

Shirai, T., \& Fukushima, T. 2001, AJ, 121, 3270

Simon, J. L., Bretagnon, P., Chapront, J., et al. 1994, A\&A, 282, 663

Standish, E. M. 1982, A\&A, 114, 297

Standish, E. M. 1998, JPL Planetary and Lunar Ephemerides, DE405/LE405, JPL IOM 312.F-98-048

Williams, J. G. 1994, AJ, 108, 711 